\title{
Éduquer à la condition terrestre
}

Francine Pellaud et Richard-Emmanuel Eastes

\section{(2) OpenEdition}

Journals

Édition électronique

URL : http://journals.openedition.org/ere/5606

DOI : 10.4000/ere.5606

ISSN : 2561-2271

Éditeur

Centr'ERE

Référence électronique

Francine Pellaud et Richard-Emmanuel Eastes, «Éduquer à la condition terrestre », Éducation relative à l'environnement [En ligne], Volume 15 - 2 | 2020, mis en ligne le 21 janvier 2021, consulté le 22 février 2021. URL : http://journals.openedition.org/ere/5606 ; DOI : https://doi.org/10.4000/ere.5606

Ce document a été généré automatiquement le 22 février 2021. 


\title{
Éduquer à la condition terrestre
}

\author{
Francine Pellaud et Richard-Emmanuel Eastes
}

1 Le développement durable (DD) a passé le cap de la trentaine sans parvenir à modifier fondamentalement les paradigmes dans lesquels évoluent nos sociétés occidentales. Les excès du capitalisme ont atteint des sommets avec la financiarisation de l'économie et les transitions écologique, énergétique ou numérique ne semblent pas pouvoir tempérer les ardeurs d'un consumérisme de plus en plus débridé.

2 L'éducation en vue d'un développement durable (EDD) a, quant à elle, coiffé SainteCatherine sans parvenir à marier les disciplines scolaires dans une approche systémique et complexe et sans permettre aux questions socialement vives d'être intégrées dans les cursus scolaires. Malgré les multiples recherches en didactique, en sociologie de l'éducation et même en neurosciences, l'école continue à enseigner " des savoirs démontés sans donner les clés qui permettent de les remonter selon des logiques autres que disciplinaires » comme l'écrivait Michel Develay en 1996 déjà, dans son livre Donner du sens à l'école. Les thématiques environnementales ne dépassent souvent pas la gestion du tri des déchets et quelques autres "éco-gestes», qui contribuent à laisser penser qu'avec un peu de bonne volonté et quelques saines habitudes, les problèmes environnementaux pourront être résolus.

Le bilan semble bien noir. Pourtant, depuis des décennies on n'avait pas vu, dans le monde occidental et même parfois au-delà une mobilisation concertée telle que celle de la jeune génération face à l'urgence climatique ${ }^{1}$. Fait nouveau, toutefois : mues par la prise de conscience du caractère mortifère de la trajectoire suivie par la civilisation thermo-industrielle occidentale (Vincent, 2019), les revendications des jeunes semblent aller non pas dans le sens d'un élargissement de leurs possibles mais dans celui de l'acceptation d'une restriction de leur confort matériel. Nous n'avons de fait jamais assisté à autant de mobilisation citoyenne, à autant d'actions individuelles ou partagées pour arriver aux objectifs sociaux et environnementaux que les gouvernements semblent bien incapables d'atteindre. 


\section{Le concept de développement durable et sa médiatisation}

4 En 1992, la Conférence de Rio validait les constats du Rapport Brundtland de 1987 et faisait du développement durable l'enjeu du siècle à venir. Le monde occidental prenait conscience que son mode de vie ne pouvait être généralisé à l'ensemble de la planète et qu'il devait, pour des raisons écologiques mais également humanistes, être repensé. D'immenses efforts de sensibilisation à l'idée de «durabilité » furent alors menés tous azimuts, notamment à travers la déclinaison de l'Agenda 21 (UN, 1992), d'abord sous sa forme «grand public " (Keating, 1992), puis sous celle d'Agendas 21 locaux (pour les régions, cantons, villes et communes qui ont alors développé leur propre Agenda), d'Agendas spécifiques à différents milieux, tels que le monde de la culture ou du sport, ainsi que d'Agendas 21 scolaires. Quant aux entreprises, leurs réflexions sur ces sujets commencèrent à s'exprimer à travers la responsabilité sociétale des entreprises (RSE).

Différentes chartes jalonnent ce parcours médiatique, telle que la Charte d'Aalborg (1994) concernant spécifiquement l'urbanisme et l'idée de "villes durables", ou la Charte des établissements et entreprises publics (2005) pour ne citer que les plus connues.

6 Mais le développement durable, c'est aussi l'occasion, pour beaucoup d'entreprises, de donner une image responsable de leurs activités. Ainsi, toutes les grandes marques de voiture font désormais référence à ce concept sur leurs sites internet et des entreprises telles qu'Air France-KLM ou Bayer ${ }^{2}$ proposent même des chartes de développement durable (Charte du développement durable, non daté). Ce faisant, elles tentent de faire reconnaître leurs efforts en matière de responsabilité sociétale ou de protection de l'environnement, alors que l'impact de leurs activités continuent à aller à l'encontre des objectifs écologiques adoptés lors des différentes Conférences des Nations Unies sur les changements climatiques (COP).

7 De tels mouvements doivent-ils être perçus comme une avancée sociétale ou, au contraire, comme une manière de "verdir» des pratiques écologiquement ou socialement irresponsables? Pour pallier ce risque, l'économie s'est, elle aussi, tournée vers différents modèles dont les intitulés, tels que "l'économie verte» (UN Environment Programme, non daté), la « chimie verte» (Anastas et Werner, 1998) ou la « croissance verte » (CEDEF, 2019), conduisent à envisager une économie capable de se prémunir du «Green washing » usuel (Canfin, 2019). Cela suffit-il à doter l'économie d'une éthique qui ne ferait plus de l'enrichissement et de la croissance l'objectif principal de son développement? Certes non. Mais pour comprendre cet état de fait, il est nécessaire de mieux cerner les visions du monde associées au concept de développement durable.

\section{Développement durable et vision(s) du monde}

8 Nous inspirant des modèles du développement durable décrits et schématisés par la Fondation suisse d'Éducation pour l'Environnement (FEE, 2012), nous pouvons identifier ici deux principales visions du développement durable : forte et faible.

Dès 1920, l'économiste Alfred Marshall, avec sa loi des rendements non-proportionnels, mettait en évidence que le capital environnemental est fini et non renouvelable (Daly et 
Cobb, 1994). Or, sans ce que nous offre la Terre, pas de possibilité de développement social et, sans ce dernier, pas d'économie. Dans cette conception dite " forte » de ce qui, à l'époque, ne s'appelait pas encore le développement durable (Figure 1), l'écologie est clairement posée comme une condition sine qua non de l'existence des sphères sociale et économique.

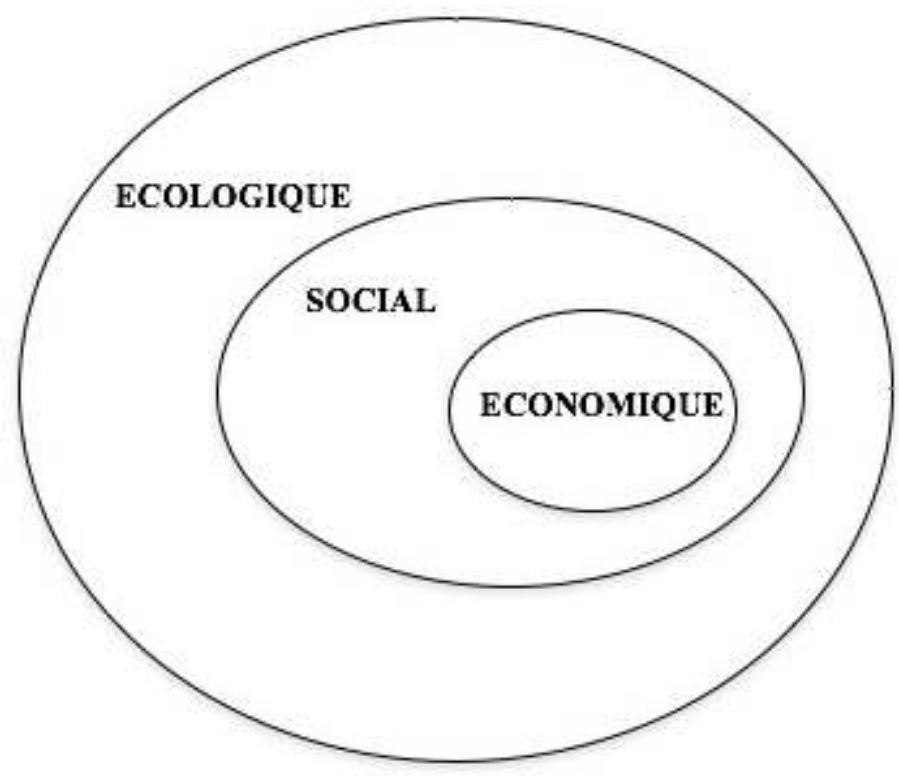

Figure 1 : Vision « forte » du développement durable

Cette thèse est reprise par l'économiste Ignacy Sachs, considéré comme un précurseur du développement durable (Criqui, 2019). Le concept d' "écodéveloppement » qu'il propose lors de la conférence des Nations Unies en 1972 à Stockholm, s'appuie sur des valeurs fortes d'équité dans la prise en charge des besoins matériels et immatériels de chaque individu, d'autonomie, respectant les contextes historiques, culturels et écologiques de toutes décisions et de prudence écologique, le développement devant se faire en harmonie avec la nature (Sachs, 1980). Si le non-gaspillage et la répartition équitable des ressources sont au cœur de sa réflexion, il ne remet toutefois pas en question l'idée de croissance, tel que le font des économistes comme Georgescu-Roegen (Grinevald, 2006) ou Serge Latouche (2006).

11 Une deuxième conception du développement durable (Figure 2), émane de l'approche économique de Hartwick (1977), où la nature n'est plus que l'un des trois piliers sur lesquels repose d'idée de durabilité (Tichit, 2005). Ancrée dans une économie libérale classique, cette conception "faible » autorise l'idée de marchandisation de la nature afin de maintenir un équilibre artificiel entre les trois sphères que sont l'économie, l'écologie et le social. 


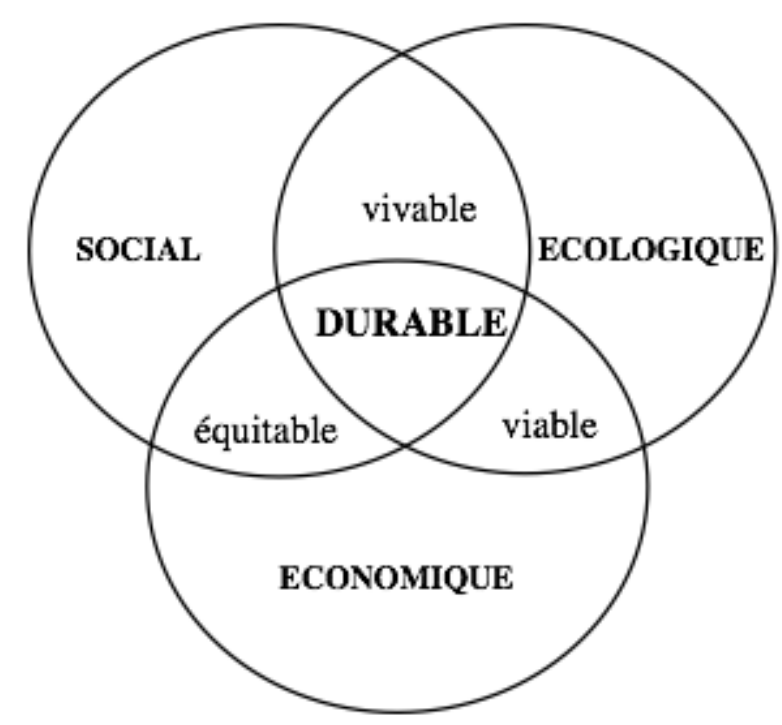

Figure 2 : Vision faible du DD

Figure 2 : Vision « faible » du développement durable

En 1987, Gro Harlem Brundtland signe le rapport de la Commission mondiale sur l'environnement et le développement de l'ONU (Brundtland, 1987) qui va ensuite fixer la notion de développement durable pour les décennies à venir. La Commission part du constat que l'économie est omniprésente et que c'est sa croissance non régulée qui conduit aux déséquilibres sociaux et aux perturbations environnementales auxquels le monde assiste. Plutôt que de récuser la primauté de l'économie, le rapport affirme l'interdépendance des trois pôles et donc la nécessité de les maintenir en équilibre.

Cette représentation faible de la durabilité est plus ancrée dans son époque que la précédente. À la fin du XXe siècle, l'enjeu essentiel était en effet de sensibiliser les milieux économiques et politiques sans les effrayer. On comprend dès lors facilement pourquoi c'est cette deuxième conception qui s'est imposée. Élaborée pour toucher le plus grand nombre, elle conduisit à une vision du monde consensuelle et donc peu militante, ne désignant aucun responsable mais définissant des responsabilités réciproques pour tous les acteurs.

\section{L'utopie du développement durable}

La vision «faible » du développement durable, selon laquelle il suffirait de combiner astucieusement les dimensions économique, sociale et environnementale du "développement humain " pour que le développement occidental de la seconde partie $\mathrm{du} \mathrm{XX}^{\mathrm{e}}$ siècle corrige ses défauts et s'étende à la planète entière, a fait long feu. Cette étonnante utopie fonde encore les 17 Objectifs de Développement Durable (ODD) « pour sauver le monde » (sic) de l'ONU (Figure 3), desquels s'inspire la construction de la majorité des Agendas 21 de la planète et, de plus en plus, les programmes scolaires, du moins en ce qui concerne la France. 


\section{(9) SUSTAINABLE GMAISA}
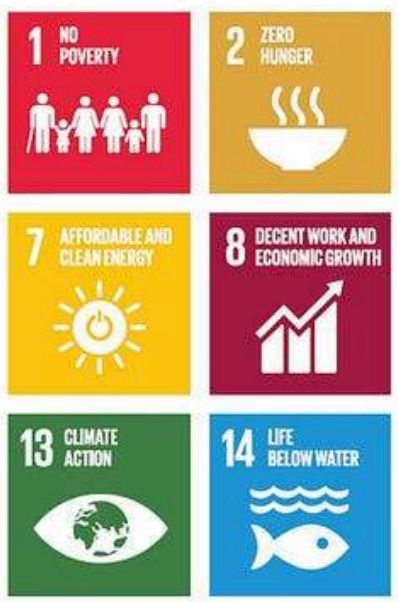
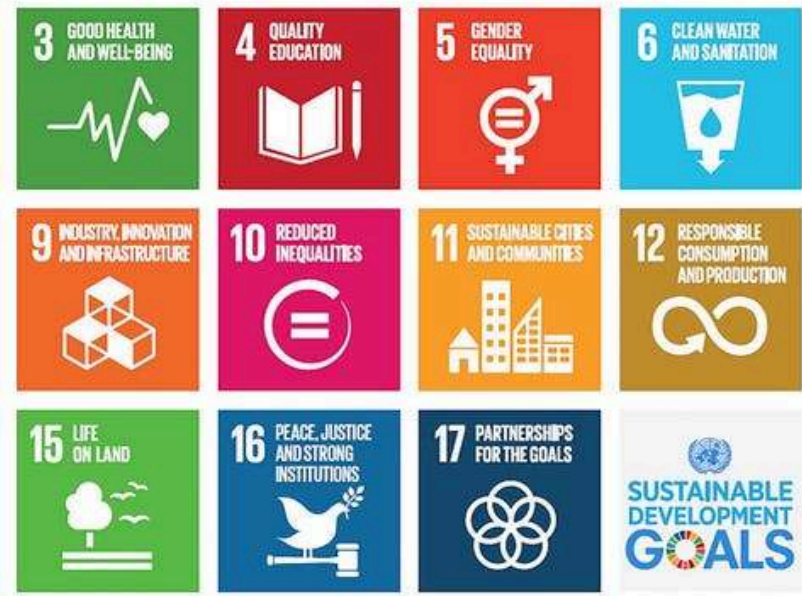

17 Pakrisersips

(4)

SUSTAINABLE

SUSTAINABLE GALS

Figure 3 : Les 17 Objectifs de développement durable (ODD) de I'ONU. Wikimedia commons.

Car, il faut bien le reconnaître, grâce à sa dénomination à la fois pléonastique et oxymorique (Eastes et Pellaud, 2013), le concept a l'immense mérite de faire accepter à une grande majorité d'acteurs la nécessité d'intégrer les questions environnementales à leurs réflexions, actions et investissements. Il laisse en effet entendre que le monde de 2030 peut être pensé comme une projection de celui du tournant du siècle, où chacun conserverait ses avantages et privilèges. La responsabilisation citoyenne, la pression de l'opinion publique sur les responsables politiques, le développement de nouvelles solutions technologiques et la signature de quelques traités internationaux auront tôt fait de régler les «quelques " externalités négatives liées à notre relation pourtant de plus en plus extravagante avec la Terre.

Pourtant, la gravité des crises environnementales auxquelles nous nous voyons soudain confrontés ne devrait-elle pas nous amener à remettre en cause cette vision du monde bâtie sur l'idée qu'un développement « durable » serait possible?

\section{Un concept sédatif}

Les stigmates de la catastrophe environnementale se creusant chaque semaine un peu plus, on est en droit de se demander si ce concept, bien que toujours mobilisateur, est encore adapté à la situation. Alors que fleurissent les notions d'anthropocène (Henneton et Confavreux, 2013) ou de capitalocène (Laurent, 2019), de collapsologie (Servigne et Stevens, 2015) et de survivalisme, il est temps de remettre en question la vision non disruptive que le "développement durable", tout autant que celle de transition écologique (Ministère de la Cohésion des territoires et de Relations avec les collectivités territoriales, 2019)33 , offre de l'avenir des Terriens et d'accepter le fait qu'à trop rassembler, il a peut-être fini par devenir un "concept-sédatif». Après être parvenu à sensibiliser à la cause environnementale ceux qui ne voyaient dans le mouvement « écolo » que des marginaux bons à élever des chèvres dans le Larzac, n'at-il pas fait de nous ce que Bruno Latour (2019) appelle des « climato-quiétistes»? Or 
quand les négateurs de la crise climatique usent de ressorts agnotologiques (Eastes, 2018) toujours plus puissants pour détourner l'attention de l'impérieuse nécessité d'opérer une transition écologique radicale, le climato-quiétisme commence fort à ressembler lui-même à du climato-scepticisme. Non par la force des convictions, mais par celle de l'inaction.

Qu'on ne s'y méprenne pas: les hérauts du développement durable, des origines du concept à nos jours, étaient des héros. Ce qu'ils ont fait était ce qu'ils pouvaient faire de mieux vu l'ampleur de la tâche et le contexte socio-politique et économique conjugué à une aspiration et à une confiance sans bornes dans le progrès scientifique et technologique. Mais les résultats escomptés ne sont pas au rendez-vous. Pour y avoir consacré une part prépondérante de leur carrière professionnelle, les auteurs de cet article n'en prennent acte qu'avec plus de tristesse. Mais plutôt que de s'enferrer dans une solution inopérante, ils ont la conviction qu'il est nécessaire de repartir et d'aller de l'avant. D'essayer autre chose. Encore et toujours.

Si nous avions l'opportunité de remonter le temps tout en conservant en mémoire les étapes qui ont conduit à l'état actuel de notre planète, que pourrions-nous tenter pour éviter d'emprunter la même trajectoire, celle dont l'inexorable propension est de nous conduire dans la direction d'un grand basculement environnemental, social, économique et (géo)politique (Severino et Ray, 2011)? Puisque nous saurions qu'elle finirait par se révéler anesthésiante, il n'y aurait pas d'autre choix que d'admettre d'emblée l'impossibilité de la continuité contenue dans l'idée de « durabilité ».

Dès lors, quelle vision programmatique de la vie sur Terre aurions-nous dû construire, il y a trente ans, pour éviter les chimères des mondes hors-sol (Latour, 2017) proposés aujourd'hui par les Trump, Bolsonaro et autres Johnson, aspirés dans une fuite en avant et un repli sur soi qui ne peuvent qu'amplifier le désastre en retardant sa prise de conscience, pour seulement quelques années de profits supplémentaires?

Changer de logiciel. Sortir du paradigme d'un bien-être et d'un mieux-vivre fondés sur l'acquisition de biens matériels. Quitter la « dictature de l'économie » (Forrester, 2000) ou celle «de la croissance» (Moreau, 2005) qui a transformé le temps en argent et poussé à l'extrême l'individualisme et la compétitivité. Reconstruire sur d'autres bases les idées d'épanouissement et de prospérité, extraire l'idée même de modernisation de son carcan scientiste et techniciste, dépasser le clivage gauche-droite désormais stérile de la plupart des politiques occidentales pour le réorienter vers la question de notre appartenance collective à la Terre et de la manière dont nous l'habitons (Latour, 2017) : autant de défis qui nécessitent de penser de nouveaux concepts pour définir notre rapport au monde, de nouveaux attracteurs politiques, une nouvelle définition de la citoyenneté, et par là même, une nouvelle éducation. Mais cette dernière ne peut être envisagée en dehors de son ancrage social et politique.

\section{Du besoin du collectif}

22 La communauté scientifique, et notamment celle des sciences humaines et sociales, est mobilisée depuis longtemps sur ces questions; en témoigne notamment l'explosion des débats scientifiques sur la pertinence de la collapsologie (Leprince, 2019) ou même celle du concept d'anthropocène (Bonneuil et Fressoz, 2013), pourtant plus fondée scientifiquement. Mais dans un monde ravagé par les atteintes environnementales, par l'explosion des inégalités et à l'aube d'une prévisible crise migratoire sans précédent, 
réfléchir aux nouvelles conditions matérielles de notre existence suppose, comme le suggère Bruno Latour (2017), d'être capables de décider collectivement "où nous voulons atterrir ", c'est-à-dire vers quelles nouvelles manières d'exister et d'habiter nos espaces de vie nous voulons nous diriger.

En parallèle, nous devons également décider de comment nous y rendre. Ce volet de la transition écologique est celui qui a été le plus exploré par les réflexions relatives aux conditions d'un développement durable, mais en négligeant la plupart des destinations qui nécessitaient une remise en question radicale des fondements de notre économie carbonée : à quoi bon isoler sa maison si l'on emprunte l'avion à chaque occasion? A quoi bon les kiwis bios s'ils proviennent de Nouvelle-Zélande lorsqu'on habite en Europe? A quoi bon les fermes solaires si c'est pour alimenter des raffineries de pétrole?

Sans compter qu'avant de savoir où aller et comment y aller, encore faut-il savoir où nous sommes (Figure 4)! Or nombreux sont les idéologues qui, n'adhérant pas aux solutions qualifiées indifféremment de bolchéviques ou d'obscurantistes (Delmas, 2019) proposées par les écologistes, préfèrent nier les causes ou minimiser l'urgence et créer du doute sur le point de départ de notre voyage, plutôt que de réfléchir à leurs responsabilités et d'admettre la nécessité des changements à opérer (Oreskes et Conway, 2012 ; Riche, 2019).

Où sommes nous ?

Prendre acte sans ambiguïté de l'état environnemental de notre planète

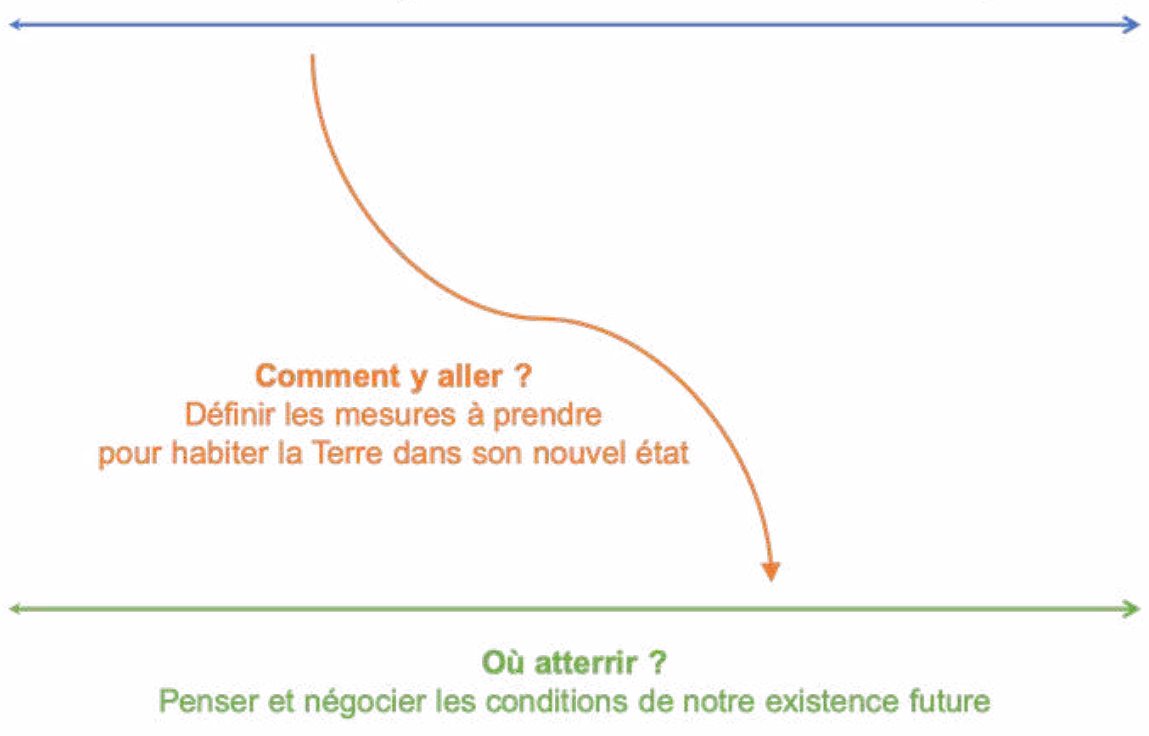

Figure 4 : Où sommes-nous ? Où atterrir ? Comment y aller ? Un voyage à penser collectivement. Note : Les significations des axes sont laissées à la libre appréciation des lecteurs.

Ces trois questions poussent à prendre acte sans ambiguïté de l'état environnemental de notre planète, à penser et négocier les conditions de notre existence future, ainsi qu'à définir les mesures à prendre pour habiter la Terre dans son nouvel état. Émerge alors immédiatement une ultime interrogation: comment organiser les modalités de ces négociations pour être collectivement en mesure de prendre des décisions admises par tous? En France, la récente crise des "gilets jaunes » a montré combien il était difficile d'y répondre. 

démocratiques adéquats pour construire réellement ensemble une vision commune de l'avenir et refonder notre conception du bien commun. À ce jour, si la diversification de figures médiatiques représentatives de différents courants écologiques - parfois contradictoires d'ailleurs (Fremeaux et Jordan, 2020) - témoigne d'une sensibilisation accrue à la crise environnementale auprès de nouvelles catégories de la population, elle conduit aussi à une polarisation sociale très préoccupante, car stérilisante, de l'action publique. Faut-il aller jusqu'à penser, comme Dominique Bourg et Kerry Whiteside (2010) que "la démocratie représentative n'est pas en mesure de répondre aux problèmes écologiques contemporains » et que "sauvegarder la biosphère exige de repenser la démocratie elle-même »?

L'objectif fondamental n'est évidemment pas de passer d'une « dictature économiste » à une « dictature écologiste ». S'accorder sur notre point de départ, décider ensemble de la destination et négocier le chemin à prendre pour s'y rendre, sachant que ce point de départ se dérobera sous nos pieds si nous ne le quittons pas et que, une fois partis, il n'y aura pas de retour en arrière possible, voilà le voyage dans lequel nous, Terriens, sommes irrémédiablement engagés. L'eau, l'air, la terre, le feu et l'ensemble du vivant figurent parmi les passagers, et il faudra désormais tenir compte de leur agentivité.

Quant à l'État, s'il est fondamental dans une optique de régulation et d'équité, sa place et son rôle doivent être également négociés afin que son intervention ne soit pas associée, de loin ou de près, à un totalitarisme. A l'instar de Criqui (2019), inventons un nouvel «Etat développeur » dont les préoccupations seront avant tout le bien commun en lieu et place du PIB.

29 Nous avons conscience que ce positionnement relève de l'utopie démocratique ; mais c'est une utopie que nous devons nous imposer avant de commencer à penser, à l'instar de l'historien narrateur de l'essai d'anticipation de Conway et Oreskes (L'Effondrement de la civilisation occidentale, 2014), que la dictature est le seul et dernier régime propre à nous protéger de notre inaction chronique.

\section{Quelle vision pour quelle éducation?}

Face à cette prise de conscience radicale, comment positionner l'éducation? Pour tenter de comprendre sur quels principes nouveaux il pourrait être possible de rebâtir une éducation à ce que nous pourrions a minima appeler la "transition écologique ", il est nécessaire d'interroger les fondements de l'actuelle éducation au développement durable (EDD).

Sans étonnement, la conception adoptée par l'école a été celle qu'ont promue, dès le rapport Brundtland, les Nations Unies et tout notre système capitaliste, à savoir la conception « faible » évoquée plus haut. Ainsi, malgré une volonté affichée du système éducatif de développer l'esprit critique et l'autonomie de pensée, ce qui a été considéré comme la conception forte du développement durable est politiquement trop en rupture avec le modèle dominant des $\mathrm{XX}^{\mathrm{e}}$ et $\mathrm{XXI}^{\mathrm{e}}$ siècle dans le monde occidental pour être adoptée - ou du moins officiellement autorisée - par et pour cette communauté éducative. La logique linéaire, pourtant simple et efficace, qui considère que si l'on protège la nature, on permet aux humains de subvenir à leurs besoins essentiels et 
donc accessoirement de développer une économie au service de l'amélioration de leur confort, apparaît donc potentiellement trop militante.

Or, en plus d'offrir deux visions du monde différentes (l'une forte et l'autre faible), le concept de développement durable peut être communiqué de manières divergentes (Eastes et Pellaud, 2013; Eastes, 2020) (Figure 5) : l'éducation qui en émane fera par exemple référence à des compétences liées à la recherche d'un idéal de civilisation dans sa posture programmatique, à la capacité à reproduire des exemples significatifs dans sa posture descriptive ou encore à des procédures ou des ensembles de normes, suivant que le concept est présenté de manière normative ou prescriptive.

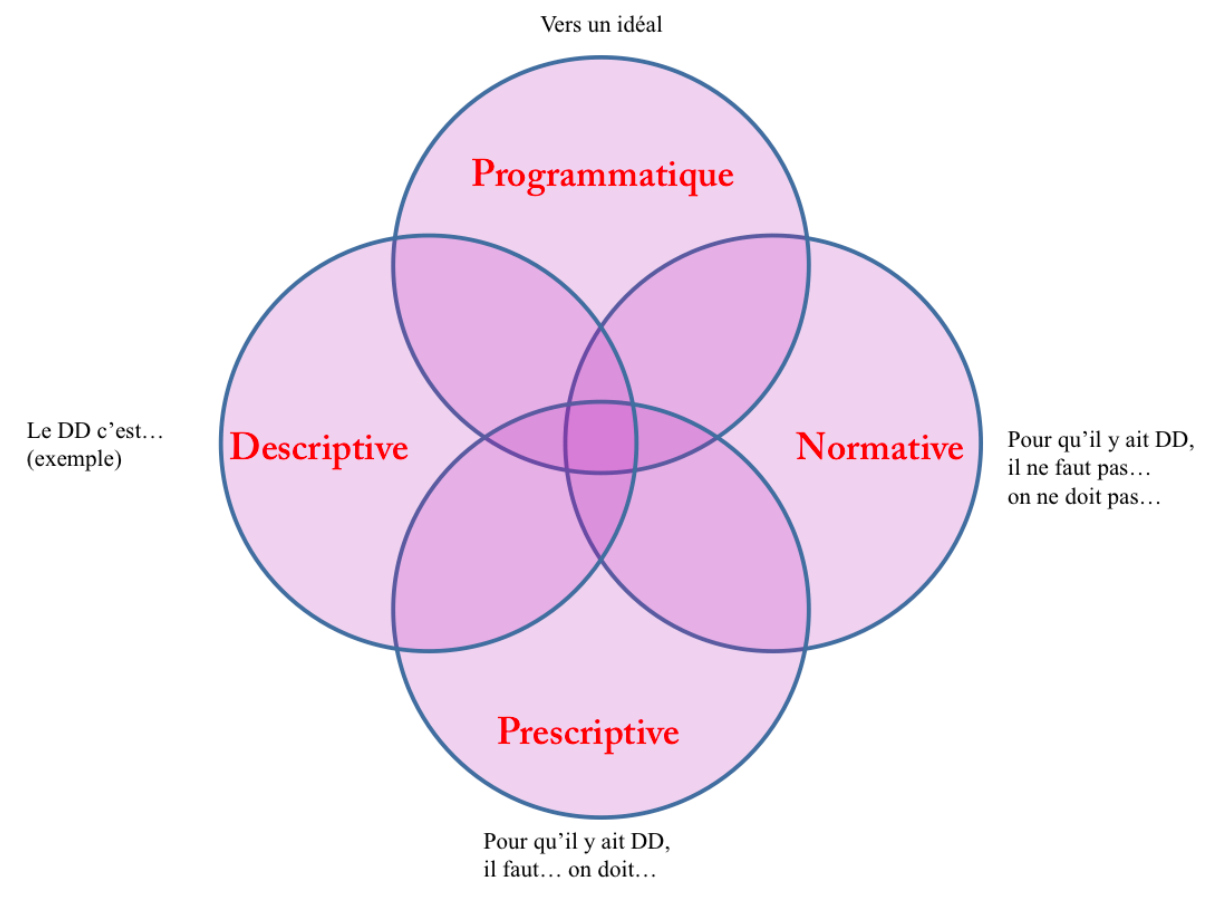

Figure 5 : Les quatre postures du DD (Eastes, Pellaud, 2013)

Figure 5 : Les quatre postures du développement durable (Eastes et Pellaud, 2013). dénomination EDD. Derrière se cachent tous les «écogestes » tels que, pour la posture prescriptive : trier les déchets, couper l'eau lorsqu'on se lave les dents, prendre des douches plutôt que des bains, éteindre les lumières lorsque l'on sort d'une pièce, etc. Et, pour la posture normative: ne pas manger de fraises en hiver, ne pas utiliser de plastique, ne pas jeter de déchets par terre, etc.

La posture descriptive est liée à des exemples de bonnes pratiques : l'organisation des transports urbains ou de la gestion des déchets dans la ville de Curitiba (Brésil), le commerce équitable selon Max Havelaar, l'agriculture intégrée, etc. Enfin, la posture programmatique est parfaitement illustrée par l'Agenda 21. Ce dernier est axé sur des recommandations et non des prescriptions, visant un idéal d'équité, de viabilité et de bien-être dans une vision idyllique d'une économie qui resterait au service de l'humain. Un tel idéal repose somme toute sur une régulation " ancestrale ", puisqu'il relève d'un « développement qui répond aux besoins du présent sans compromettre la capacité des générations futures à répondre à leurs propres besoins » (Brundtland, 1987), ce qu'ont 
réussi à faire à peu près toutes les générations qui ont précédé la nôtre. Un idéal pas complètement abouti toutefois, puisqu'inspiré par une perspective anthropocentriste peu consciente de l'inscription de l'humanité dans le plus vaste réseau du vivant.

Les compétences que l'on souhaite développer à travers ces différentes postures relèvent d'une bonne capacité d'imitation, voire d'adaptation dans l'approche descriptive, ainsi que d'une soumission à l'ordre établi dans les approches normative et prescriptive. Dans l'approche programmatique, elles portent davantage sur une compréhension des mécanismes de la complexité, complétée - dans un idéal éducatif par une pensée prospective créative afin de sortir du "faire mieux » dont on connaît les limites, pour entrer dans le « faire autrement ».

On comprend facilement pourquoi cette dernière approche n'a jamais réussi à franchir les grilles des cours d'école. Au-delà des difficultés que suggère une telle éducation, elle vise le développement d'individus capables d'une pensée indépendante, capable de s'affranchir des normes et des limites prescrites pour remettre en question l'existant... jusqu'à peut-être remettre en question le paradigme même du développement durable. Est-ce vraiment là le souhait des dirigeants, des industriels et plus encore, des lobbies en place?

37 Cette conception «faible » du développement durable et plus spécifiquement ses approches normatives et prescriptives ont favorisé une intégration des préoccupations environnementales dans les programmes éducatifs européens depuis les années 2000. Un bienfait en matière de sensibilisation, sans doute, mais une telle conception du monde a certainement contribué à promouvoir une vision angélique d'une civilisation occidentale qui n'aurait qu'à « faire un peu moins mal » pour pouvoir continuer à vivre comme avant.

\section{Penser le basculement... et s'y adapter}

Ainsi, l'éducation concernant ces questions doit se réinventer d'urgence. Or s'il convient de préparer les citoyens de ce monde à endiguer les extraordinaires crises environnementales, politiques, économiques et sociales à venir, il y a deux manières de le faire: en enseignant à nos enfants les connaissances et compétences qui leur permettront de prévenir et résoudre ces crises, ou en leur prodiguant celles qui leur permettront de s'y adapter. Préparer la résilience plutôt que le changement, c'est s'acheminer vers un douloureux constat d'échec. Ne serait-il pas temps de leur apprendre également à penser le monde d'après et à y vivre, voire à y survivre, s'il devait s'avérer impossible de le construire autrement?

Les réponses à ces questions sont éminemment politiques. Dès lors, penser une éducation en vue d'une transition écologique radicale est-elle possible sans faire entrer au sein de l'école des visions du monde qui seront inévitablement accusées d'être " orientées idéologiquement »? C'est d'ailleurs bien ce que ses opposants reprochent à Greta Thunberg, l'accusant soit de manipulation politique, soit de préparer la " dictature verte ». Pourtant, l'école est déjà idéologiquement orientée - simplement par le fait de ne pas traiter de ces questions et de propager la vision d'un modèle de développement qui nous détruit - mais cela, trop peu le reconnaissent. Un militantisme qui dicterait les conditions d'une transformation radicale de nos sociétés, autant que les approches prescriptive ou dogmatique décrites précédemment, contient les germes d'un endoctrinement soumettant l'élève à une volonté qui lui serait supérieure. Qu'il 
s'agisse d'une vision, dans le cas du militantisme, ou d'un règlement, voire de « lois ", formalisées ou non, dans le cas du prescriptif-normatif, l'élève « suit » un modèle, entre dans un "moule", sans forcément qu'il y ait de sa part une décision consciente. Le risque de tomber dans un despotisme ou une société du «prêt-à-penser » est grande. "L'enfer est pavé de bonnes intentions", dit-on, et ces visions, aussi justes et bienveillantes qu'elles puissent être sont susceptibles de participer à une sorte de propagande, enlevant la part de responsabilité de l'acteur.

Car la tentation est grande, lorsque nous sommes face à une urgence telle que nous la vivons aujourd'hui, d'imposer des idées, des gestes, des façons de faire ou de penser, des lois qui interdisent, voire qui punissent les contrevenants à un mouvement qui nous semble "juste». "La fin détermine les moyens" pour reprendre une autre expression populaire.

41 Si la « fin » pour le développement durable peut en effet prendre la forme de gestes et d'habitudes salutaires pour la planète, l'éducation doit conserver des objectifs plus ambitieux et plus nobles en matière de compétences, de compréhension et d'autonomie de pensée. Il y a une différence énorme entre un élève qui répète des gestes simplement parce qu'il a assimilé ce qu'on attendait de lui, et un élève qui choisit délibérément de les accomplir parce qu'il en a compris le bien-fondé et estime que c'est de son devoir, de sa responsabilité de les adopter. Politiquement, le premier élève s'inscrit dans une vision totalitaire, le second dans une vision démocratique où l'opinion éclairée et la liberté d'expression et de pensée sont respectées.

Pourtant, au vu de la gravité de la situation environnementale, l'entrée d'une certaine radicalité écologique à l'école nous semble indispensable. Mais jusqu'où déplacer le curseur? Quels verrous sommes-nous prêts à faire sauter? Quels garde-fous devronsnous préserver quoiqu'il arrive?

Quelles que soient les réponses à ces questions, il est une valeur qui ne saurait être négociable: l'école devra garantir des objectifs d'autant plus élevés en matière de compétences, de compréhension et d'autonomie de pensée qu'elle se verra chargée d'orienter la société vers une transition plus radicale. Pour justement ne pas tomber dans le despotisme écologique.

\section{Participer aux changements}

Comment, dans ces conditions, inventer un courant éducatif qui succédera à l'éducation au développement durable? Pour y réfléchir, nous nous inspirons des questions fondamentales de Bruno Latour (2017) présentées précédemment et proposons une grille d'analyse susceptible de clarifier quelque peu les pistes à suivre dans la perspective du "grand basculement " évoqué plus haut. Elle reprend les trois questions: Où sommes-nous? Où voulons-nous aller (Bruno Latour dirait «Où atterrir?») ? Comment pourrions-nous y aller?

À la première question "Où sommes-nous?" - et contrairement à ses habitudes consistant à n'enseigner que des connaissances stabilisées depuis des décennies -, l'école se doit de faire preuve de courage en intégrant les données scientifiques environnementales produites récemment. Quitte à les réviser ou à revenir en arrière, mais en se montrant intraitable face aux tentatives de désinformation climatosceptiques qui gangrènent les médias sociaux, jusqu'aux sites d'information pour enseignants ${ }^{4}$. L'approche épistémologique des sciences offre à cet effet des pistes 
tout à fait intéressantes. Grâce à une meilleure compréhension de la façon dont les différentes sciences se construisent, de leurs méthodes et des critères de scientificité qu'elles adoptent et qui les définissent (Popper, 1963), mais en mettant également en lumière leurs errances et leurs doutes, la manière dont elles gèrent l'incertitude ou les probabilités, dont elles permettent de démasquer les impostures complotistes et les failles qui peuvent apparaître dans les arguments proposés par les dénialistes, et notamment par les détracteurs du travail réalisé par le Groupe Intergouvernemental sur l'Évolution du Climat (GIEC).

Parallèlement, et comme l'éducation au développement durable le préconisait déjà mais certes sans grand succès, elle doit développer encore plus largement l'enseignement de la pensée systémique et complexe (Morin, 1999; 2000) : mettre en évidence non plus la linéarité mais les interdépendances, les rétroactions, la relativité, la gestion du flou, de l'aléatoire, de l'incertain (Pellaud, 2011, 2017). Pour littéralement « changer le logiciel » de la manière dont nous enseignons aux élèves à penser le monde (Pellaud, 2019 ; Pellaud et coll. 2019).

À la deuxième question "Où voulons-nous aller?", l'école doit donner aux futurs citoyens que sont ses élèves la capacité d'élaborer collectivement la réponse. Aux compétences systémiques évoquées ci-dessus, elle ajoutera la pensée prospective et créatrice (Pellaud, 2013) et visera le développement d'individus capables d'une pensée autonome et critique, mais surtout collaborative. Plusieurs compétences émotionnelles sous-tendent cette dernière. La confiance en soi, l'empathie, le respect de l'altérité ou le partage, pour ne citer que les plus évidentes (Pellaud et Gay, 2017). Car la difficulté principale ne résidera pas dans le fait de répondre à cette question mais, comme nous l'avons vu, de mettre en place des conditions permettant d'y répondre. Et, pour ce faire, nous devons miser sur des êtres humains épanouis, bénéficiant d'une confiance en eux les rendant responsables de leurs choix de vie (Pellaud et coll. 2020) et dont l'objectif ultime n'est pas le pouvoir (souvent économique) individuel. Là encore, l'école actuelle permet-elle le développement de tels individus?

Il faudra enfin peut-être laisser un peu de côté la troisième question "Comment y aller?", pourtant favorite d'une éducation au développement durable fondée sur des propositions destinées à faire évoluer nos modes de vie. Parce qu'il est vain de chercher à décider du chemin et du véhicule si l'on ne sait ni d'où on part, ni où on va. On remplacera alors les approches prescriptive et normative de l'EDD par une approche plus programmatique, non plus fondée sur des «écogestes » mais visant à développer de véritables compétences.

\section{Pour « une éducation à la condition terrestre »}

En 2011, nous affirmions que l'éducation au développement durable avait pour mission " d'insuffler un changement d'état d'esprit pour " voir plus loin », anticiper sur l'avenir et agir en conséquence » (Pellaud, 2011). En ce sens, elle entendait déjà préparer une hypothétique transition écologique, même si le terme était encore peu populaire. Cette définition reste pour nous d'actualité, mais elle nécessite désormais de prendre acte du caractère inévitable de la catastrophe, comme nous y incite le philosophe Jean-Pierre Dupuy (2004) depuis quinze ans déjà, ainsi que de l'échéance extrêmement courte que les scientifiques ont identifiée avant que nous basculions définitivement dans un effondrement irréversible. À ce stade, et alors que la pertinence du concept de 
«transition » est elle-même déjà contestée (Fressoz, 2018), même une « éducation à la transition écologique » nous semble devenue insuffisamment forte. Alors quoi ?

l'école démocratique où l'opinion et la liberté d'expression et de pensée sont respectées : tel est le défi que nous proposons à la communauté éducative de relever pour fonder une nouvelle forme d'action. Au-delà du développement durable, de la transition écologique, de l'écocivisme et de la préservation de l'environnement, l'éducation qu'il convient de bâtir devra être soucieuse de construire une société capable de définir démocratiquement les moyens de protéger radicalement des humains les biens communs que sont la biosphère et son enveloppe atmosphérique, en prenant acte des limites de la Terre : une éducation relative à la condition terrestre.

51 Pour y parvenir, l'acquisition des connaissances nécessaires à la compréhension des événements actuels reste indispensable. Mais elle doit être menée dans un climat de confiance face à la capacité de l'être humain à relever les défis que les multiples changements environnementaux vont lui imposer. Pour ce faire, il est nécessaire, comme le propose Jacques Dubochet (2020), «d'inventer un narratif d'un autre bonheur, qui remplacera l'ancien, morne et ennuyeux, que l'on croit être le seul possible car c'est le seul que l'on connait ». Un narratif qui nous conduise vers des valeurs, et surtout vers un besoin de valeurs (Pellaud et Gay, 2017), tournées définitivement vers l'être et non vers l'avoir ou le paraitre. Vers une valorisation du collectif où la complémentarité l'emporterait sur la compétition et où la liberté individuelle rimerait avec principe de responsabilité (Jonas, 1979).

Ce travail sur les imaginaires collectifs est l'un des axes thématiques du nouveau Centre interdisciplinaire de durabilité $\left(\mathrm{CID}^{5}\right)$ de l'Université de Lausanne. Leur projet de recherche "Futurs possibles", tente de "stimuler le dialogue entre sciences et fiction autour $d u$ nécessaire renouveau des imaginaires du futur face aux urgences climatiques.» Dans le monde scolaire, ce travail d'anticipation, basé sur une clarification des valeurs (Pellaud, 2011), est proposé à travers l'utilisation de contes dont les élèves doivent inventer la fin (Pellaud et Muths, 2006 ; Pellaud et Robach, 2010) en fonction de choix éthiques. Il serait intéressant de vérifier si de telles pratiques contribuent à la création de ce nouvel imaginaire collectif et de cette capacité à se projeter dans cette appartenance à un système Terre dont nous ne pouvons pas nous extraire et dont l'avenir ne dépendra, en fin de compte, que de notre aptitude à le maintenir dans un équilibre dynamique.

\section{Et la formation des enseignant $\cdot$ es ?}

En ce qui concerne la question de la posture enseignante, sept universités européennes ont travaillé, dans le cadre du projet «A Rounder Sense of Purpose » en collaboration avec Erasmus+, à définir les compétences que devraient posséder les enseignant.e.s pour arriver à une éducation au développement durable. Une matrice, proposée en 2011 déjà par l'United Nations Economic Commission for Europe (UNECE), sert de base aux travaux réalisés au sein de ce projet. Sans toucher au fond des propositions, nous avons adapté certaines formulations afin de mieux faire ressortir la proximité entre ces propositions et les différents principes de la pensée complexe et les finalités que nous proposons depuis près de 20 ans dans notre propre approche de l'EDD (Pellaud, 2000) (Tableau 1).

Éducation relative à l'environnement, Volume 15 - 2 | 2020 
Tableau 1 : Les compétences des enseignant·es en EDD.

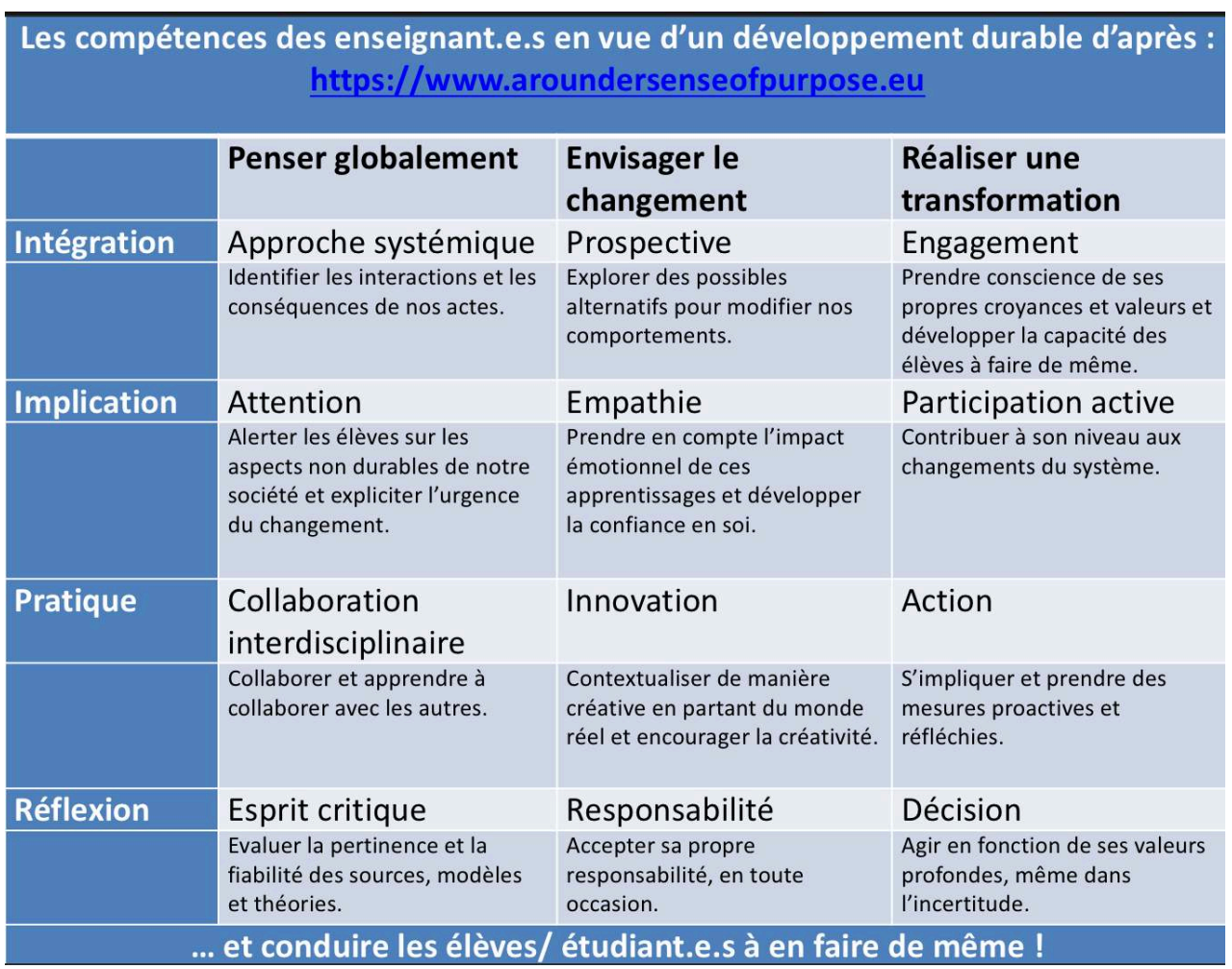

Mais ce qui nous paraît essentiel et fondamentalement innovant réside dans la posture de l'enseignant.e. En effet, il/elle se doit d'alerter les élèves et les étudiant.es sur l'urgence climatique, sur les conséquences de nos choix de sociétés. Ils/elles doivent être proactifs en s'impliquant dans des actions réfléchies et en exemplifiant leurs décisions par des actions régies en fonction de leurs valeurs. Ainsi, clarifier sa posture, assumer ses choix font partie de notre responsabilité. Aujourd'hui, il est criminel de se taire, autant que d'induire une attitude défaitiste et catastrophiste chez nos élèves. Susciter l'inquiétude peut éveiller une "solastalgie » ou une "éco-anxiété » comme celle qui est à la source même de l'engagement de Greta Thunberg (Véronique, 2019). Mais l'éco-anxiété n'est toutefois pas garante d'un investissement écologique ou du moins, en faveur d'une réduction des impacts négatifs de l'humain sur son environnement. Au contraire, elle peut contribuer à une attitude totalement opposée qui peut se résumer ainsi : "Puisque tout est foutu, ça ne sert à rien de se priver ", que l'on entend d'ores et déjà dans les cours de récréation.

Enfin, sans nier les risques potentiels de la situation actuelle, notre présent est-il si parfait qu'il ne puisse être amélioré ? Sommes-nous si heureux, enfermés dans nos peurs, nos paradigmes et une confiance aveugle en une technoscience salvatrice? Une société plus solidaire, plus respectueuse de l'autre au sens le plus large, moins axée sur les biens matériels et la promotion sociale ne pourrait-elle pas apporter un bien-être et un épanouissement que notre monde occidental peine à (re)trouver? La sobriété volontaire, le bénévolat, l'entre-aide, le partage d'un travail dans lequel nous pourrions nous épanouir parce que participant de manière positive à un monde moins cupide et égocentré, ne pourrait-il pas remplacer avantageusement les "like» de nos réseaux sociaux? 
En invoquant l'idée "d'éducation à la condition terrestre " telle que nous l'avons définie plus haut, nous faisons le pari qu'il est encore possible d'atteindre un idéal sociétal par le développement des intelligences sociale, émotionnelle et cognitive. Un dernier sursaut d'optimisme avant de confier nos destins à l'effondrement et à l'état d'urgence.

\section{BIBLIOGRAPHIE}

Anastas, P. T. et Warner, J. C. (1998). Green Chemistry: Theory and Practice, New York, Oxford University Press. A rounder sens of purpose. Consulté sur www.aroundersenseofpurpose.eu Bonneuil, C. et Fressoz, J-B. (2013). L'évènement anthropocène, Paris : Édition Le Seuil Bourg, D. et Whiteside, K. (2010). Vers une démocratie écologique. Paris : Edition Le Seuil.

Brundtland, G. H. (1987). Notre avenir à tous. Rapport de la Commission mondiale sur l'environnement et le développement de l'ONU. Consulté sur https://fr.wikisource.org/wiki/

Notre_avenir_\%C3\%A0_tous_-_Rapport_Brundtland

Canfin, P. (2019). La taxonomie européenne est indispensable pour financer la transition. Novethic, 17 décembre 2019. Consulté sur www.novethic.fr/actualite/finance-durable/isr-rse/lataxonomie-europeenne-est-indispensable-pour-financer-la-transition-selon-pascalcanfin-148019.html

Centre de documentation Économie Finances : un service ouvert à tous (CEDEF) (2019). Le portail de l'Economie, des Finances, de l'Action et des Comptes. Consulté sur www.economie.gouv.fr/ cedef

Conway, E-M. et Oreskes, N. (2014). L'effondrement de la société occidentale. Paris : Éditions Les Liens qui libèrent.

Charte d'Aalborg (1994). Consulté sur www.iufn.org/wp-content/uploads/2013/08/1-FR-ChartedAalborg.pdf

Charte développement durable des établissements publics et des entreprises publiques (2005). Consulté sur www.ecologique-solidaire.gouv.fr/sites/default/files/

CDDEP_charte_developpement_durable_des_etablissements_et_des_entreprises_publics.pdf

Charte du développement durable AirFrance et KLM. (non daté). Consulté sur https:// procurement.af-klm.com/fr/images/charte_DD_FR_mai2012_tcm1105-762891.pdf

Ministère de la cohésion des territoires et des relations avec les collectivités locales (2019). Transition écologique. Consulté sur www.cget.gouv.fr/thematiques/transition-ecologique

Daly, H. E. et Cobb, J. B. (1994). For the Common Good. Boston : Beacon Press

Delmas, J-L. (2019). Pourquoi Greta Thunberg suscite-t-elle tant d'hostilité ? 20 minutes planète, le 22 juillet 2019. Consulté sur www.20minutes.fr/planete/2569119-20190722-pourquoi-gretathunberg-suscite-tant-hostilite 
Develay, M. (1996). Donner du sens à l'école. Paris : ESF, collection « Pratiques \& enjeux pédagogiques ".

Dubochet, J. (2020). Table ronde : Ces jeunes qui refusent la démission du monde. Les Artisans de la Transition. Théâtre de Vidy, 7 janvier 2020. Lausanne.

Dupuy, J-P. (2004). Pour un catastrophisme éclairé. Paris : Éditions Points

Eastes, R-E. et Pellaud, F. (2013). Les malentendus du développement durable. Revue francophone du développement durable 2, 83-95

Eastes, R-E. (2018). La science face à la production des ignorances. Club 44. Consulté sur https:// vimeo.com/275614069

Eastes, R-E. (2020). Peut-on définir... l'école, le développement durable, la science, la médiation scientifique? Savoirs en Société. Consulté sur : www.youtube.com/watch ?v=_04D9GOVlIs

Frommherz, C. et Monnet, A. (2012). Modèles du développement durable. Fondation suisse d'Education pour l'Environnement (FEE), Bulletin éducation environnement.ch 1/2012, Annexe. Consulté sur www.education21.ch/sites/default/files/uploads/pdf_fr/Publications/ annexe_eech_1_2012.pdf

Forrester, V. (2000). Une étrange dictature. Paris : Édition Fayard

Fremeaux, I. et Jordan, J. (2020). Quelle culture voulons-nous nourrir ? Revue Terrestres. Consulté sur www.terrestres.org/2020/08/04/quelle-culture-voulons-nous-nourrir/

Fressoz, J-B. (2018). Transition, piège à con ? Le Média. Consulté sur www.youtube.com/watch ? $\mathrm{v}=100 \mathrm{r} 5 \mathrm{O} 4-2 \mathrm{wU}$

Grinevald, J. (2006). « Nicholas Georgescu-Roegen, dissident de l'Occident et visionnaire de la décroissance ", dans La Revue Durable no 20, avril-mai-juin 2006, p. 8-13.

Henneton, T. et Confavreux, J. (2013). L'apocalypse et l'anthropocène. Vacarme no 65, 202-233

Jonas, H. (1979). Le principe responsabilité. Paris : Éditions Flammarion

Latouche, S. (2006). Le Pari de la décroissance. Paris : Éditions Fayard

Latour, B. (2017). Où atterrir ? Comment s'orienter en politique. Paris : Éditions La découverte.

Laurent, A. (2019). Climat : Sommes-nous dans le « capitalocène »? Média Usbek \& Rica, 20 janvier 2019. Consulté sur : https://usbeketrica.com/article/climat-sommes-nous-dans-le-capitalocene

Leprince, C. (2019). Théorie de l'effondrement : la « collapsologie » est-elle juste une fantaisie sans fondement ? France Culture. Consulté sur www.franceculture.fr/ecologie-et-environnement/ theorie-de-leffondrement-la-collapsologie-est-elle-juste-une-fantaisie-sans-fondement

Morin, E. (1990). Introduction à la pensée complexe. Paris : Édition ESF.

Morin, E. (2000). Les Sept Savoirs nécessaires à l'éducation du futur. Paris : Éditions Le Seuil.

Oreskes, N. et Conway, E. (2012). Les Marchands de doute. Paris : Éditions Le Pommier.

Pellaud, F. et Robach, C. (2010). L'Ogresse. Paris : Éditions Les Atomes Crochus.

Pellaud, F et Muths, D. (2006). Plus loin que le bout de son nez... Les Cahiers Pédagogiques no 443, 45-46.

Pellaud, F. (2000). L'utilisation des conceptions du public lors de la diffusion d'un concept complexe, celui de développement durable, dans le cadre d'un projet en muséologie. Thèse de doctorat no 287 , Université de Genève. Consulté sur https://archive-ouverte.unige.ch/unige :97 
Pellaud, F. (2011). Pour une éducation au développement durable. Paris : Éditions Quae.

Pellaud, F. (2013) Anticiper sur l'avenir pour agir en conséquence : les besoins en matière de pensée créatrice-prospective. Consortium EDD de la COHEP. Consulté sur www.education21.ch/ sites/default/files/uploads/cohep/contributions/Pellaud \%20_Pensee_prospective.pdf

Pellaud, F. (2017). Changements climatiques et transition énergétique : complexité, approche systémique et cartes conceptuelles. Revue francophone du développement durable 9, 99-111.

Pellaud, F. et Gay, P. (2017) Des connaissances au passage à l'acte : les complexités de l'éducation au développement durable. Revue francophone du développement durable, Hors-série 5, 6-17.

Pellaud, F. (2019). Quels objectifs pour une éducation en vue d'un développement durable ? Dans M. Graner et A. Giordan, Ecole changer de cap, dossier thématique : L'éducation à l'environnement et au développement durable (p. 15-26). Consulté sur www.ecolechangerdecap.net/spip.php? article466\&lang $=\mathrm{fr}$

Pellaud, F., Heinzen, S., Marbacher, V., Brumeau, Q., Quartenoud, A. et Sautaux, M., (2019). «FABuleux objets ». Un projet interdisciplinaire mêlant éducation au développement durable, éthique et travaux manuels : que peut-il apporter aux étudiants, futurs enseignants primaires ? Dans M. Graner et A. Giordan, Ecole changer de cap, dossier thématique : L'éducation à l'environnement et au développement durable (p. 42-49). Consulté sur http://www.ecolechangerdecap.net/IMG/pdf/ dossier_ecole_ddurable.pdf

Pellaud, F., Gay, P., Blandenier, G., Massiot, P. et Dubois, L. (2020). Developing Self-Confidence through the transformation of evaluation practices. Dans A. Diemer, E. Nedelciu, M. Schellens, M. Morales et M. Oostdijk, Paradigms, Models, scenarios and practices for strong sustainability (397-406). Clermont-Ferrand : Éditions Oeconomia.

Popper K. (1963 - Traduction française, 1985). Conjectures et réfutations, La Croissances du savoir scientifique. Paris : Payot.

Riche, A. (2019). Climat : les marchands de doute se font plus pernicieux. Le Soir.be, 8 octobre 2019. Consulté sur : https://www.lesoir.be/252318/article/2019-10-08/climat-les-marchands-dedoute-se-font-plus-pernicieux

Sachs, I. (1980). Partie I - Approches de l'écodéveloppement. Dans Sachs, I., Stratégies de l'écodéveloppement (15-35). Ivry-sur-Seine : Éditions de l'Atelier.

Servigne, P. et Stevens, R. (2015). Comment tout peut s'effondrer. Paris : Éditions Le Seuil.

Severino, J-M. et Ray, O. (2011). Le grand basculement. Paris : Édition Odile Jacob

Tichit, A. (2005). Le développement durable. Ressources en sciences économiques et sociales. Lyon : École normale supérieure de Lyon, Ressources en sciences économiques et sociales. Consulté sur http://ses.ens-lyon.fr/articles/ariane-tichit-le-developpementdurable-25383\#section-0

UN (1992). Action 21. Consulté sur www.un.org/french/ga/special/sids/agenda21/

UNenvironment programme (2020). Green economy. Consulté sur www.unenvironment.org/ explore-topics/green-economy

Véronique, P. (2019). Angoisse, dépression...L'éco-anxiété, l'autre effet du réchauffement climatique. L'Express, 8 août 2019. Consulté sur www.lexpress.fr/actualite/societe/ environnement/angoisse-depression-l-eco-anxiete-l-autre-effet-du-rechauffementclimatique_2093702.html 
Vincent, C. (2019). Climat : les nouveaux récits de l'écologie. Le Monde, 3 janvier 2019. Consulté sur www.lemonde.fr/idees/article/2019/01/03/les-nouveaux-recits-de-1-

ecologie_5404756_3232.html

\section{NOTES}

1. Friday for Future (2019). Grève mondiale pour le climat. Consulté sur https:// fr.globalclimatestrike.net/

2. Consulté sur Bayer AG (2020). Sustainabilit, www.bayer.com/en/sustainability.aspx

3. Voir à ce propos la remise en question de l'idée même de transition : Fressoz, J-B. (2018).

4. Le site Médiachimie (http://www.mediachimie.org/ressource/le-changement-climatiquequestion-encore-ouverte propose des ressources aux enseignantes et nous pouvons y lire, sous la plume de Vincent Courtillot (détracteur déclaré de l'origine anthropique du changement climatique), un court texte intitulé : Le changement climatique : question encore ouverte ? La conférence plénière d'introduction à la Maison de la Chimie prononcée par ce même auteur y est aussi disponible. Vincent Courtillot est également l'un des membres fondateurs du site climatosceptique des « Climato-Réalistes » (https://http://www.climato-realistes.fr/

5. Centre Interdisciplinaire de Durabilité (CID) de l'Université de Lausanne, https://news.unil.ch/ display/1569937206204

\section{RÉSUMÉS}

Si le développement durable a réussi l'exploit de s'immiscer jusqu'au sein de l'économie et que son éducation a investi la plupart des programmes scolaires, force est de constater que, près de 30 ans plus tard, extinctions massives, pollutions, disparités sociales, catastrophes écologiques et surexploitations sont notre pain quotidien. Ce résultat, contre-productif et alarmant, n'est-il pas à attribuer à un concept qui a endormi les consciences plutôt que de les réveiller ? Dès lors quelles sont les questions fondamentales qui doivent guider notre réflexion en tant que penseurs de l'éducation? Quelle est notre place face à une urgence qui ne peut plus se satisfaire de beaux discours?

Although sustainable development has succeeded in penetrating the economy, and most school curricula incorporate education on the subject, almost 30 years later, it is clear that mass extinction, pollution, social disparities, ecological disasters and overexploitation are our daily fare. Can this counterproductive and alarming result possibly be attributed to a concept that has appeased our consciences, rather than appealing to them ? Against this backdrop, what are the fundamental questions that should guide our reflection as the thinkers of education? What is our role in the face of an emergency that calls for more concrete action than words alone? 


\section{INDEX}

Mots-clés : éducation au développement durable, éducation à la condition terrestre, urgence climatique, transition écologique, visions du monde, démocratie.

Keywords : education for sustainable development, education on the state of the earth, climate emergency, ecological transition, world visions, democracy.

\section{AUTEURS}

\section{FRANCINE PELLAUD}

Francine Pellaud, docteure en sciences de l'éducation, professeure à la Haute école pédagogique de Fribourg en Suisse, enseigne l'éducation au développement durable et la didactique des sciences. Auteur de contes, de spectacles et de concepts muséographiques autour des sciences et du développement durable, elle développe actuellement des ressources pédagogiques destinées à favoriser la pensée complexe à travers une pédagogie interdisciplinaire portant sur des questions socialement vives (https://blog.hepfr.ch/transformations/). Ces ressources alimentent ses recherches.

\section{RICHARD-EMMANUEL EASTES}

Richard-Emmanuel Eastes, agrégé de chimie, docteur en sciences de l'éducation et en philosophie, auteur de plusieurs ouvrages sur les relations science-société, est actuellement chercheur associé au STS Lab de l'Université de Lausanne, responsable du centre de soutien à l'enseignement supérieur pour les 28 hautes écoles de la Haute Ecole Spécialisée de Suisse Occidentale (HES-SO) et directeur de la société de conseil en communication scientifique et en ingénierie cognitive Segallis. 